\title{
BMJ Open Effects of yoga on well-being and healthy ageing: study protocol for a randomised controlled trial (FitForAge)
}

\author{
Josefine Östh, ${ }^{1}$ Vinod Diwan, ${ }^{2}$ Maria Jirwe, ${ }^{3,4}$ Vishal Diwan, ${ }^{5}$ Anita Choudhary, ${ }^{6}$ \\ Vijay Khanderao Mahadik, ${ }^{6}$ Michaela Pascoe, ${ }^{7}$ Mats Hallgren ${ }^{1}$
}

To cite: Östh J, Diwan V, Jirwe M, et al. Effects of yoga on well-being and healthy ageing: study protocol for a randomised controlled trial (FitForAge). BMJ Open 2019;9:e027386. doi:10.1136/ bmjopen-2018-027386

- Prepublication history and additional material for this paper are available online. To view these files, please visit the journal online (http://dx.doi. org/10.1136/bmjopen-2018027386).

Received 23 October 2018

Revised 9 April 2019

Accepted 10 April 2019
Check for updates

(C) Author(s) (or their employer(s)) 2019. Re-use permitted under CC BY-NC. No commercial re-use. See rights and permissions. Published by BMJ.

For numbered affiliations see end of article.

Correspondence to Dr Mats Hallgren; mats.hallgren@ki.se

\section{ABSTRACT}

Introduction Due to ageing populations worldwide, the burden of disability is increasing. It is therefore important to develop interventions that improve healthy ageing, reduce disability onset and enhance life quality. Physical activity can promote healthy ageing and help maintain independence, yet many older adults are inactive. Yoga is a form of physical activity that aims to improve health and may be particularly suitable for older adults. Research indicates positive effects of yoga on several health-related outcomes; however, empirical studies examining the benefits of yoga on well-being among the elderly remain scarce. This study protocol reports the methodology for a 12-week yoga programme aimed to improve health and well-being among physically inactive older adults. Methods and analysis Three group parallel, single-blind randomised controlled trial. Two comparison groups are included: aerobic exercise and a non-active wait-list control. In total, 180 participants aged 65-85 years will be recruited. Assessments will be performed at baseline and postintervention (12-week follow-up). The primary outcome is subjective well-being. Secondary outcomes include physical activity/sedentary behaviour, mobility/ fall risk, cognition, depression, anxiety, mood, stress, pain, sleep quality, social support and cardiometabolic risk factors. Data will be analysed using intention-to-treat analyses, with mixed linear modelling.

Ethics and dissemination This study is approved by the Ethical Review Board in Stockholm (2017/1862-31/2). All participants must voluntarily agree to participate and are free to withdraw from the study at any point. Written informed consent will be obtained from each participant prior to inclusion. Results will be available through research articles and conferences. A summary of key results will be publicly available through newspaper articles.

Trial registration number DRKS00015093, U1111-12174248

\section{BACKGROUND}

Populations are ageing globally, leading to new public health challenges. Within this demographic transition, it is estimated that the number of people aged $\geq 60$ yearswill increase from 900 million in 2015 to 1400 million by $2030,{ }^{1}$ thus increasing the prevalence of non-fatal disabilities such as

\section{Strengths and limitations of this study}

- This is the first randomised controlled trial to explore the effects of yoga on well-being compared with a physically active and inactive control group among elderly adults.

- Inclusion of both active and inactive control groups enables comparison of the effects on several levels, including indirect effects of the yoga intervention.

- All exercise will be free of charge, instructor-led and undertaken at established fitness centres, decreasing the risk of adverse events.

- Generalisability of study findings may be limited by a healthy entrant bias.

- Subjective measurements of physical activity could overestimate activity levels.

mental, behavioural and musculoskeletal disorders. ${ }^{2}$ Many of the commonly experienced health problems among the elderly can be prevented or delayed by embracing a physically active lifestyle ${ }^{1}$; research in elderly populations shows that regular physical activity can benefit mood disorders, cognitive deficits, pain, mobility and increase independence. $^{3}$

Despite these known benefits, levels of inactivity tend to increase with age. ${ }^{4}$ Approximately $30 \%-60 \%$ of adults aged $\geq 60$ years across the WHO regions do not meet the recommended activity levels ${ }^{4}$; that is, $\geq 150$ min of moderate-intensity or $75 \mathrm{~min}$ of vigorous-intensity aerobic physical activity (or an equivalent combination) per week. ${ }^{5}$ Activity levels among younger adults are showing a similar trend; in 2016, more than one-third of adults were inactive according to recent WHO global estimates based on self-reported data ( $\mathrm{n}=1.9$ million; 168 countries) ${ }^{6}$ Thus, relatively little progress has been made to reduce global inactivity levels ${ }^{6}$ which has major health impacts on non-communicable diseases (NCDs) ${ }^{7}$ and constitutes the fourth leading risk factor for mortality. ${ }^{5}$ 
A recent review indicates that older adults are interested in and able to engage in physical activity interventions. ${ }^{8}$ For many, physical activity is a means to enhance wellbeing and functional abilities that help maintain independence and life quality. ${ }^{9}$ Well-being is a multidimensional concept which includes physical, psychological and social components ${ }^{10}$ and low ratings of subjective well-being are associated with higher prevalence of chronic disease and lower life quality among the elderly. ${ }^{11}$ Similarly, activity levels are shown to predict subjective well-being among older adults ${ }^{12-14}$ which in turn is associated with healthy ageing and longevity. ${ }^{11}$

Yoga is an increasingly popular form of physical activity $^{15}$ and refers to a unity of the body, mind and spirit. ${ }^{16}$ Recent research indicates beneficial effects of yoga on several health outcomes affecting elderly adults, including balance and mobility, ${ }^{17-19}$ cardiometabolic health, ${ }^{20-23}$ cognition, ${ }^{24-26}$ sleep quality and quality of life. ${ }^{27}$ Yoga practice has also been associated with better subjective well-being ${ }^{28-30}$ and improved mental health outcomes such as depression and anxiety. ${ }^{31}{ }^{32}$ With few exceptions, however, ${ }^{1952733}$ the evidence base supporting yoga for older adults consists of exploratory, feasibility or pilot studies, often with small sample sizes and methodological weaknesses. Hence, there is a need for rigorously designed and adequately powered community-based trials of yoga which include assessments of diverse health outcomes.

\section{Rationale}

While aerobic exercise is recommended for the prevention of several NCDs, ${ }^{7}$ yoga's emphasis on flexibility and balance may help to reduce the risk of fall-related injuries, ${ }^{17-19}$ which are prevalent among older adults and a leading cause of injury-related hospitalisation. ${ }^{34}$ Healthy ageing does not merely correspond to longevity, but also optimising well-being. ${ }^{11}$ With a holistic focus that includes physical, mental and spiritual well-being, yoga could be a suitable and appealing health maintaining activity for adults who find aerobic exercise difficult or impractical. ${ }^{35}$ While previous trials have compared the effects of yoga with non-active controls, ${ }^{36}$ none have compared the effects of yoga on subjective well-being to aerobic training and a non-active (wait-list) condition among older adults.

\section{Study objective}

This controlled trial aims to examine the effects on wellbeing of a 12-week yoga intervention, focused mainly on physical postures and movements, among healthy but physically inactive Swedish adults aged 65-85 years. Results will be compared with an equivalent duration aerobic exercise programme and wait-list control group.

\section{Research questions}

- What are the effects of a 12-week yoga intervention on subjective well-being in older adults, compared with aerobic exercise and non-active wait-list control?
- Can yoga improve health outcomes that are highly prevalent among older adults?

- Does yoga participation affect total physical activity levels or sedentary behaviours?

- Does yoga affect cognitive functioning in older adults?

\section{Hypotheses}

We hypothesise that (1) compared with aerobic training, participation in yoga will be associated with small magnitude improvements in self-reported well-being, and equivalent magnitude improvements on all secondary outcomes; (2) compared with non-active control, yoga will be associated with moderate magnitude improvements on all outcomes.

\section{Trial design}

The protocol is drafted in accordance with Standard Protocol Items: Recommendations for Interventional Trials $^{37}$ (see Research checklist).

We propose to conduct a three-group parallel, singleblind randomised controlled trial (RCT). Assessments will be performed at baseline and after 12 weeks (see figure 1, participant flowchart based on the Consolidated Standards of Reporting Trials guidelines for transparent reporting of trials $^{38}$ ).

\section{METHODS}

\section{Study setting}

The study will take place in Stockholm County at established fitness centres (FCs) with several facilities throughout Stockholm. The coordination and assessments of outcomes will be performed at the Department of Public Health Sciences, Karolinska Institutet.

\section{Eligibility criteria}

To be eligible for this study, participants must be between 65 and 85 years of age, have residence in Stockholm County, be fluent in Swedish and be willing to participate.

\section{Exclusion criteria}

- Physical disability, disease or injury that could interfere with or be worsened by physical activity (eg, paralysis, inability to sit, stand and/or walk, severe pain, obstructive disease, glaucoma) or a severe cognitive deficit (eg, dementia). Severe cognitive deficiency will be assessed based on two questions: 'Do you have troubles with your memory that affects your daily life?' and 'Do you have a diagnosis for dementia?' Cognition will be measured as a secondary outcome, using verbal fluency (see Secondary outcomes, below). For this assessment, we are guided by the mean values in Tallberg $e t a l^{39}$ and will exclude those with values $<21.5$ for word fluency, 12.1 for animal fluency and 8.5 for verb fluency.

- Extensive surgery the past year, or planned the coming year.

- Insulin-dependent diabetes (those with fasting venous blood glucose levels $\geq 5.6 \mathrm{mmol} / \mathrm{L}$ will be advised to 


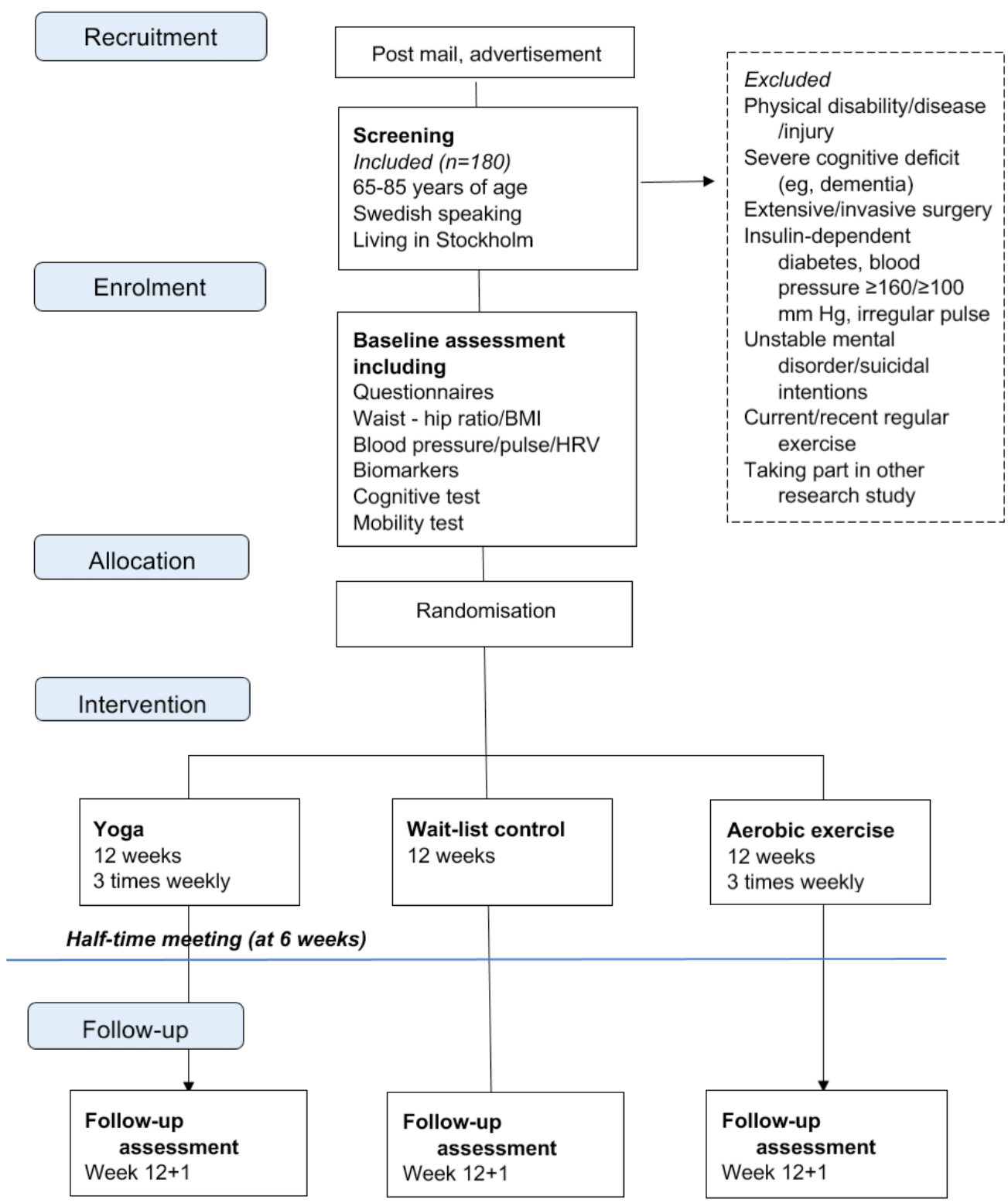

Figure 1 Participant flowchart for parallel design, based on the CONSORT guidelines ${ }^{38}$ for transparent reporting of trials. BMI, body mass index; CONSORT, Consolidated Standards of Reporting Trials; HRV, heart rate variability.

seek advice at their local primary healthcare centre but can still participate), blood pressure $\geq 160 \mathrm{~mm} \mathrm{Hg}$ systolic, $\geq 100 \mathrm{~mm} \mathrm{Hg}$ diastolic (blood pressure will be measured in sitting position twice in each arm) or irregular pulse.

- Advised not to participate in moderate exercise by their doctor.

- Recently diagnosed with serious mental illness (eg, psychosis or bipolar disorder) or indicating acute signs of suicidality (eg, recent and ongoing thoughts of death/suicide).

- Currently or recently (past 3 months) engaged in regular structured exercise, on average 2 days/week or more.

- Simultaneously taking part in another research study.

\section{Interventions \\ Yoga}

Participants randomised to the yoga group will receive a free 3-month membership at a FC offering supervised group senior yoga classes for older adults with the aim of improving flexibility, core strength and balance. The 60-min classes (also available to adults not part of the study) are considered suitable and safe for older adults and beginners. Classes will be offered at multiple locations and several times during the week using a standardised format to reduce the risk of intervention variability. Participants will meet a personal trainer (PT) before commencing yoga classes, encouraged to attend classes three times a week during the intervention period and to perform yoga at home at those occasions they 
cannot make it to the FC. The length of the intervention is consistent with other yoga-based interventions for older adults. ${ }^{15}$

The senior yoga intervention is based on Hatha yoga, which consists of gentle physical postures (asanas), plus breathing exercises and limited meditation. Hatha yoga is considered particularly suitable for older adults. ${ }^{40}$ The opening postures include cat/cow pose, mountain pose and partial sun salute. Thereafter, classic Hatha yoga postures are instructed, including chair pose, plank, tree pose, warrior I and II, downward facing dog, side stretch, spine twist, cobra pose and bridge pose. The finishing series includes relaxation exercises; dead man's pose and relaxation. ${ }^{41}$ A detailed description of these movements can be found elsewhere. ${ }^{40}$ Classes will be delivered by qualified yoga instructors employed at the FC.

\section{Aerobic exercise}

Participants in the aerobic exercise group will receive a free FC membership for 3 months and advised to participate in supervised group aerobic training classes suitable for older adults. All classes are offered by the same FCs providing the yoga intervention. Classes will include cycling/spinning, indoor/Nordic walking, dancing and aerobics. Participants will undertake aerobic exercise three times per week for 60 min each session and complete a weekly exercise diary. If the participants cannot make it to the FC three times weekly, they will be prompted to exercise at home. Thus, the location, frequency and duration of aerobic exercise will be matched with the yoga group. Participants will meet a PT before commencing exercise. All exercise classes will be delivered by qualified fitness instructors employed at the FC. Classes will also be available to adults not taking part of the study.

\section{Wait-list}

Participants randomised to the non-active comparison group will be wait-list controls. At inclusion, they will be instructed to continue their daily activities as usual (eg, without engaging in regular structured exercise). They will complete an exercise diary at the end of the 12-week study. As an incentive, these participants will receive a FC card valid for 3 months when they attend their postintervention assessment.

\section{Measures to maintain adherence}

Participants will be recruited based on their address and proximity to the FC to minimise travel time and encouraged to contact a research assistant to discuss any issues relating to study adherence. Scheduled meetings will be preceded with text messages or phone calls 1 day before. Moreover, all participants in the two physical activity arms will receive one $30 \mathrm{~min}$ face-to-face sessions with a qualified PT; at the beginning of the intervention. The purpose of the PT meeting is to make the participants familiar with the FC, the booking system and to make an exercise plan for the 12 weeks based on the prerequisites of each participant in order to minimise the risk of injury. Evidence suggests that supported exercise interventions lead to better adherence than non-supported interventions. ${ }^{42}{ }^{43}$ Participants will complete a weekly exercise diary and attendance at the yoga and aerobic classes will be recorded electronically when participants enter the FCs. All classes will be offered free of charge and all study participants will be offered a $20 \%$ discount on full year membership at the FC after completion of their postintervention assessments.

\section{Outcome variables}

Primary outcome variable

- Subjective well-being will be measured using the short version of Life Satisfaction Index, version $\mathrm{Z}^{44-46}$; a 13-item self-administered questionnaire that aims to evaluate well-being and healthy ageing using five core components; zest, resolution and fortitude, congruence between desired and achieved goals, positive self-concept and mood tone. ${ }^{47}$

- The 5-item Satisfaction with Life Scale measuring global judgments of one's life satisfaction ${ }^{48} 49$ will also be used to assess subjective well-being.

\section{Secondary outcome variables}

- Physical activity and sedentary behaviour will be subjectively assessed using the 4-item International Physical Activity Questionnaire (IPAQ) ${ }^{5051}$ and the 5-item Simple Physical Activity Questionnaire. ${ }^{52}$

- Mobility and fall risk will be objectively assessed using the 14-item Berg Balance Scale-a validated measure of balance ${ }^{5354}$ and fall risk. ${ }^{55}$

- The verbal fluency test will objectively assess executive function, attention and information processing speed ${ }^{39}$ including categorical and letter fluency. ${ }^{56}$

- Self-reported depression will be assessed by the 20-item Geriatric Depression Rating Scale. ${ }^{5758}$

- Self-reported anxiety will be assessed using the 20-item State-Trait Anxiety Inventory Y2. ${ }^{59} 60$

- The Profile of Mood States Questionnaire (POMS) will be used to assess mood and psychological distress. ${ }^{61} 62$ The 30-item POMS includes six subscales; tensionanxiety, vigour-activity, fatigue-inertia, depressiondejection and confusion-bewilderment.

- Subjective stress (past month) will be assessed by the 10-item Perceived Stress Scale. ${ }^{6364}$

- The Brief Pain Inventory short form (9-items) will be used to assess pain severity and how pain interferes with daily life..$^{666}$

- Sleep quality during the past 2 weeks will be measured using the 7-item self-reported Insomnia Severity Index. ${ }^{6768}$

- The 9-item version of the Interview Schedule for Social Interactions short form will examine the availability of the social relations. ${ }^{69} 70$

- Blood pressure, resting heart rate, body mass index, waist-hip ratio, heart rate variability, complete blood count, blood glucose and blood lipids and saliva cortisol will be collected to assess cardiometabolic risk factors. 
- Borg rating of perceived exertion scale will be used to assess exercise intensity. ${ }^{71}$ It will be handed out to the participants in the two physical activity arms at halftime and assessed by the participant immediately after three different exercise sessions.

The abovementioned measures of life satisfaction, physical activity, sedentary behaviour, depression, anxiety, mood, stress, pain, sleep quality and social support are validated in Swedish.

\section{Sample size}

The sample size calculation is based on the effect size seen in a previous 6-month RCT comparing the effects of beginners Iyengar yoga with light walking. ${ }^{19}$ Using this study as a guide, a standardised mean difference (effect size) of around 0.2 on the primary study outcome (well-being) is anticipated, favouring the yoga group compared with aerobic training. Based on these parameters, with $80 \%$ power, two-tailed significance for the primary between-group comparisons (yoga vs aerobic exercise), an expected drop-out rate of $20 \%{ }^{72}$ and 1:1:1 allocation, a total sample of 180 participants is estimated.

\section{Recruitment}

Participants will be recruited by postal invitation and newsletter advertisements. A list of potentially eligible participants will be randomly generated by Statistics Sweden, based on age and residential status (eg, proximity to the gym, living within $3 \mathrm{~km}$ distance of the FC). Recruitment will occur during the first year of the trial in three waves. Those interested in participating will contact a research assistant who, after a brief telephone screening, will arrange a meeting to evaluate eligibility.

\section{Randomisation and blinding}

A statistician (external to the study) will generate a randomisation list using a random number computer programme. The list will be kept confidential and will only be unblinded in the event of participant withdrawal or adverse events. The allocation sequence will be transferred into separate sealed envelopes by a research assistant not part of the study team. These envelopes will be opened by research assistants together with the participant immediately following the baseline assessment. Treatment allocation will not be concealed from study participants nor the study coordinator. The study participants will be requested not to discuss their treatment allocation at follow-up meetings, when a different research assistant blinded to group allocation will be responsible for collecting data. Allocation will be kept confidential and only unblinded in the event of participant withdrawal or adverse events.

\section{Data collection}

Baseline assessment

The baseline assessment will be performed by trained research assistants prior to randomisation. The assessment covers demographic characteristics and collection of primary and secondary outcomes including anthropometric measurements, questionnaires and functional ability tests of mobility and cognition (see the Outcome variables section). Participants will receive referral for biological sampling at any of the Karolinska University laboratories and will receive a scheduled time for follow-up. Baseline assessments will take $120 \mathrm{~min}$.

\section{Postintervention assessments}

Each participant will be invited to a brief meeting of maximum $60 \mathrm{~min}$ with a research assistant at half-time (6 weeks) to discuss progress and to assess any possible

\begin{tabular}{|c|c|c|c|c|c|c|c|}
\hline \multicolumn{8}{|c|}{ STUDY PERIOD $\longrightarrow$} \\
\hline TIME POINT & & & & Enrolment & Baseline & Intervention & Endpoint \\
\hline & & & & week -1 & week 0 & week 1-12 & week $12+1$ \\
\hline Eligibility screen & & & & $x$ & & & \\
\hline Informed consent & & & & $\mathrm{X}$ & & & \\
\hline Allocation & & & & $\mathrm{X}$ & & & \\
\hline \multicolumn{8}{|l|}{ INTERVENTION } \\
\hline Yoga intervention & & & & & & $\longrightarrow$ & \\
\hline Aerobic exercise & & & & & & $\longrightarrow$ & \\
\hline Wait-list control & & & & & & & Get fitness card \\
\hline Study outcomes & Method for assessment & Subjective & Objective & & & & \\
\hline Demographics & Baseline Questionnaire & $\mathrm{x}$ & & & $\mathbf{x}$ & & \\
\hline Well-being & Life satisfaction index Z/Satisfaction with Life Scale & $\mathrm{x}$ & & & $x$ & & $x$ \\
\hline Physical activity & International Physical Activity Questionnaire & $\mathrm{X}$ & & & $\mathbf{x}$ & & $\mathrm{x}$ \\
\hline Sedentary behaviour & Simple Physical Activity Questionnaire & $\mathrm{X}$ & & & $\mathrm{X}$ & & $x$ \\
\hline Mobility/fall risk & Berg Balance Score & & $\mathbf{x}$ & & $\mathbf{X}$ & & $\mathbf{x}$ \\
\hline Cognition & Verbal fluency test & & $x$ & & $\mathrm{x}$ & & $x$ \\
\hline Cardiometabolic risks & Blood pressure, resting heart rate, BMI, WHR, Heart rate variability & & $\mathbf{X}$ & & $\mathbf{X}$ & & $\mathbf{x}$ \\
\hline - Blood tests & Complete blood count, blood lipds, blood glucose & & $\mathrm{X}$ & & $\mathrm{X}$ & & $\mathrm{x}$ \\
\hline Depression & Geriatric Depression Rating Scale & $\mathbf{x}$ & & & $\mathbf{X}$ & & $\mathbf{x}$ \\
\hline Anxiety & State-Trait Anxiety Inventory Y2 & $x$ & & & $\mathrm{x}$ & & $x$ \\
\hline Mood & Profile of Mood States & $\mathbf{x}$ & & & $\mathbf{x}$ & & $\mathbf{x}$ \\
\hline Stress & Perceived Stress Scale, saliva cortisol & $x$ & $x$ & & $x$ & & $x$ \\
\hline Pain & Brief Pain Inventory & $\mathbf{X}$ & & & $\mathbf{X}$ & & $\mathbf{X}$ \\
\hline Sleep quality & Insomnia Severity Index & $x$ & & & $\mathrm{X}$ & & $x$ \\
\hline Social support & Interview Schedule for Social Interactions short form & $\mathbf{X}$ & & & $\mathbf{X}$ & & $\mathbf{X}$ \\
\hline
\end{tabular}

Figure 2 Schedule of enrolment, interventions and assessments, according to SPIRIT $2013^{37}$ guidelines. BMI, body mass index; SPIRIT, Standard Protocol Items: Recommendations for Interventional Trials; WHR, waist-hip ratio. 
adverse events associated with the intervention. Postintervention assessments of primary and secondary outcomes will be performed at $12+1$ weeks (see Figure 2). This visit will take $\sim 120 \mathrm{~min}$.

\section{Data management}

Collected data will be kept confidential in participant case report forms (CRFs) and later transferred into electronic form (Microsoft Excel 2013). The principal investigator $(\mathrm{MH})$ is responsible for maintenance of safely kept records and back-up of data. Statistical analyses will be performed using SPSS V.22.0.

\section{Statistical methods}

Quantitative data will be collected and intention-to-treat analyses will be performed to examine effects of the yoga intervention, aerobic exercise active comparison group and the wait-list controls on the primary study outcome. A subanalysis will also be performed to investigate the effect of the recruitment cluster on the results. Missing data will be replaced using multiple imputation methods. The effects will be explored using mixed linear modelling, where group by time interactions will be reported with regression estimates, CIs and effect sizes (Hedges' g). Sensitivity analyses will be performed. Moderating effects of gender and age will be examined, plus interaction effects of education level for cognitive outcomes.

\section{Patient and public involvement}

As the study consists of a non-clinical sample recruited from the general population, patients were not involved in the development of the trial protocol. The study design was informed by (1) expertise within the research group, which consists of researchers with experience in the design and evaluation of physical activity interventions for diverse groups, and (2) the existing literature describing yoga interventions for adults and elderly populations. Results from blood and saliva tests will be offered to individual participants at follow-up. All participants will complete a brief, semistructured interview at follow-up to assess their satisfaction with the trial. Study findings will be disseminated through scientific articles and through a layman's summary published in the participating FC magazine, and on the FitForAge study website (to be established).

\section{Assessment of harms}

Trained research assistants will screen all potential participants and exclude those with health-related problems that might hinder participation or be worsened by physical activity. All adverse events will be recorded, evaluated and followed-up by the research assistants on a specific form in the CRF. Throughout the study, any adverse events needing medical care will be appropriately referred. Adverse events will be formally assessed at halftime (6 weeks) and at postintervention ( $12+1$ weeks), and participants will be instructed to inform the study coordinator of any experienced adverse events throughout the study. Decision of participant or study discontinuation will be taken by the principal investigator.

\section{Time plan}

Planned recruitment start: January 2019. Planned study end: January 2021.

\section{DISCUSSION}

Increasing physical activity levels within community settings is a significant public health priority. Physically inactive older adults who are considering a new exercise regime require updated information about the relative benefits and risks of different physical activities. This community-based RCT will explore the effects on health and well-being of a 12-week yoga intervention among physically inactive older adults (65-85 years). These effects will be compared with aerobic exercise, which is recommended for general health, and wait-list control. The physical activity programmes will be structured and supervised at established FCs in Stockholm, which will facilitate replication in other settings.

Interventions involving yoga are generally considered safe $^{73}$; adverse events are rare but may include muscle strains and soreness. ${ }^{73}$ To reduce these risks, all exercise sessions will be designed for older adults and delivered by qualified instructors and PTs with experience in physical activity prescription.

Potential strengths of the study are the inclusion of two comparison groups which will enable the effects of yoga to be compared with an active and inactive control condition. Active comparison groups should, whenever possible, control for indirect effects of the intervention, such as social interaction, which could also influence the study outcome. ${ }^{74}$ Here, the aerobic exercise group will match the yoga group on potential moderators such as the duration and location of activity, social contact and frequency of exercise. Another potential strength is the use of standardised measures and blinded follow-up assessments, which minimises assessment bias. The primary study outcome, subjective well-being, is gaining more importance in ageing research, and is shown to be closely linked to health and quality of life in elderly populations. ${ }^{11}$ Some possible limitations are also acknowledged. Study generalisability may be affected to some extent by a 'healthy entrant effect' where only those individuals with positive attitudes or expectations towards physical activity express interest in participating. Subjective measures of physical activity tend to overestimate activity levels in adults ${ }^{75}$; however, the IPAQ is a commonly used and validated measure of activity, enabling comparisons across studies.

If findings demonstrate that yoga is effective in improving well-being, mobility, mood and cognition, it may have public health implications in terms of disease prevention and treatment. Although yoga is familiar to many people, its prescription for health conditions remains rare, in part due to the current lack of empirical support for its use. If appropriately disseminated, study 
findings could inform both individual and clinical decisions around exercise prescription for older adults. The study will also deepen current knowledge of yoga's effects on health among older adults generally.

\section{ETHICS AND DISSEMINATION Consent to participate}

Every potential participant will be informed about the study aims and procedures by a research assistant prior to participation, verbally and in writing. Confidentiality, voluntariness and freedom to withdraw from the study at any point will be stated. Written, informed consent will be obtained from all participants by a research assistant (online supplementary file 1).

\section{Confidentiality}

Study participants will receive a unique study-ID at inclusion, based on the time of inclusion. Collected data will be coded and data will be stored in the CRF with the same ID which is considered source data. Collected data will be handled only by authorised people and will be directly reported into the participant CRF, kept in a locked archive at the Department of Public Health Sciences, Karolinska Institutet. The CRFs will be kept for 10 years after study end according to the standard guidelines at the Karolinska Institutet. Trial treatment randomisation codes will likewise be maintained confidential and codes broken only in case of adverse events or decision on discontinuation.

\section{Data statement}

The research protocol will be available at the institution's website; https://ki.se/en/phs/intervention-researchat-the-epicss-group. Primary results and datasets will be available from the corresponding author on reasonable request. Results will be reported on group-level only.

\section{Dissemination policy}

The results of the study will be publicly available in scientific media through research articles and conferences. A summary of key results will be publicly available for study participants and for lay people through newspaper articles.

\section{Author affiliations}

${ }^{1}$ Department of Public Health Sciences, Karolinska Institutet, Stockholm, Sweden ${ }^{2}$ Department of Public Health Sciences, Karolinska Institutet, Solna, Sweden ${ }^{3}$ Department of Health Promoting Sciences, Sophiahemmet Hogskola, Stockholm, Sweden

${ }^{4}$ Department of Neurobiology, Care Sciences and Society, Karolinska Institutet, Stockholm, Sweden

${ }^{5}$ Department of Public Health and Environment, International Centre for Health Research, Ruxmaniben Deepchand Gardi Medical College, Ujjain, Madhya Pradesh, India

${ }^{6}$ Department of Physiology, Ruxmaniben Deepchand Gardi Medical College, Ujjain, Madhya Pradesh, India

${ }^{7}$ Institute for Health and Sport, Victoria University, Melbourne, Victoria, Australia

Contributors $\mathrm{MH}$ and VinD conceived and designed the study. MH and JÖ wrote the first draft of the protocol - all co-authors contributed to subsequent revisions. JÖ is the project coordinator.
Funding This study is supported by Swedish Research Council for Health, Working Life and Welfare (FORTE), grant number 2017-00024 awarded to MH. The funding body will play no role in the design and/or conduct of the study.

Competing interests None declared.

Patient consent for publication Obtained.

Ethics approval The study is approved by the Regional Ethics Committee in Stockholm (Regionala Etikprövningsnämnden, number 2017/1862-31/2, amendment number 2018/2156-32).

Provenance and peer review Not commissioned; externally peer reviewed.

Open access This is an open access article distributed in accordance with the Creative Commons Attribution Non Commercial (CC BY-NC 4.0) license, which permits others to distribute, remix, adapt, build upon this work non-commercially, and license their derivative works on different terms, provided the original work is properly cited, appropriate credit is given, any changes made indicated, and the use is non-commercial. See: http://creativecommons.org/licenses/by-nc/4.0/.

\section{REFERENCES}

1. World Health Organization. Multisectoral action for a life course approach to healthy ageing: draft global strategy and plan of action on ageing and health. Geneva: WHO, 2016. http://apps.who.int/gb/ ebwha/pdf_files/WHA69/A69_17-en.pdf?ua=1. (Accessed 5 Sep 2018).

2. Vos T, Flaxman AD, Naghavi M, et al. Years lived with disability (YLDs) for 1160 sequelae of 289 diseases and injuries 1990-2010: a systematic analysis for the Global Burden of Disease Study 2010. Lancet 2012;380:2163-96.

3. Paterson DH, Warburton DE. Physical activity and functional limitations in older adults: a systematic review related to Canada's Physical Activity Guidelines. Int J Behav Nutr Phys Act 2010;7:38.

4. Hallal PC, Andersen LB, Bull FC, et al. Global physical activity levels: surveillance progress, pitfalls, and prospects. Lancet 2012;380:247-57.

5. World Health Organization. Global recommendations on physical activity for health. Geneva: WHO, 2010. http://www.who.int/ dietphysicalactivity/factsheet_recommendations/en/. (Accessed $9 \mathrm{Jul}$ 2018).

6. Guthold R, Stevens GA, Riley LM, et al. Worldwide trends in insufficient physical activity from 2001 to 2016: a pooled analysis of 358 population-based surveys with 1.9 million participants. Lancet Glob Health 2018;6:e1077-86.

7. Lee IM, Shiroma EJ, Lobelo F, et al. Effect of physical inactivity on major non-communicable diseases worldwide: an analysis of burden of disease and life expectancy. Lancet 2012;380:219-29.

8. Devereux-Fitzgerald A, Powell R, Dewhurst A, et al. The acceptability of physical activity interventions to older adults: A systematic review and meta-synthesis. Soc Sci Med 2016;158:14-23.

9. Franco MR, Tong A, Howard K, et al. Older people's perspectives on participation in physical activity: a systematic review and thematic synthesis of qualitative literature. Br J Sports Med 2015;49:1268-76.

10. Centers for Disease Control and Prevention. Health-related quality of life (HRQOL): well-being concepts. 2016 https://www.cdc.gov/hrqol/ wellbeing.htm\#eight (Accessed 23 Aug 2018).

11. Steptoe A, Deaton A, Stone AA. Subjective wellbeing, health, and ageing. Lancet 2015;385:640-8.

12. Lampinen $P$, Heikkinen RL, Kauppinen M, et al. Activity as a predictor of mental well-being among older adults. Aging Ment Health 2006;10:454-66.

13. Garatachea N, Molinero O, Martínez-García R, et al. Feelings of well being in elderly people: relationship to physical activity and physical function. Arch Gerontol Geriatr 2009;48:306-12.

14. Olsson LA, Hurtig-Wennlöf A, Nilsson TK. Subjective well-being in Swedish active seniors and its relationship with physical activity and commonly available biomarkers. Clin Interv Aging 2014;9:1233-9.

15. Cramer H, Lauche R, Dobos G. Characteristics of randomized controlled trials of yoga: a bibliometric analysis. BMC Complement Altern Med 2014;14:328.

16. Pandurangi AK, Keshavan MS, Ganapathy V, et al. Yoga: past and present. Am J Psychiatry 2017;174:16-17.

17. Youkhana S, Dean CM, Wolff M, et al. Yoga-based exercise improves balance and mobility in people aged 60 and over: a systematic review and meta-analysis. Age Ageing 2016;45:21-9.

18. Zettergren KK, Lubeski JM, Viverito JM. Effects of a yoga program on postural control, mobility, and gait speed in community-living older adults: a pilot study. J Geriatr Phys Ther 2011;34:88-94. 
19. Oken BS, Zajdel D, Kishiyama S, et al. Randomized, controlled, six-month trial of yoga in healthy seniors: effects on cognition and quality of life. Altern Ther Health Med 2006;12:40-7.

20. Barrows JL, Fleury J. Systematic review of yoga interventions to promote cardiovascular health in older adults. West $J$ Nurs Res 2016;38:753-81.

21. Pascoe MC, Bauer IE. A systematic review of randomised control trials on the effects of yoga on stress measures and mood. $J$ Psychiatr Res 2015;68:270-82.

22. Bowman AJ, Clayton RH, Murray A, et al. Effects of aerobic exercise training and yoga on the baroreflex in healthy elderly persons. Eur $J$ Clin Invest 1997;27:443-9.

23. Santaella DF, Devesa CR, Rojo MR, et al. Yoga respiratory training improves respiratory function and cardiac sympathovagal balance in elderly subjects: a randomised controlled trial. BMJ Open 2011;1:e000085.

24. Gothe NP, McAuley E. Yoga and Cognition: A Meta-Analysis of Chronic and Acute Effects. Psychosom Med 2015;77:784-97.

25. Gothe NP, Kramer AF, McAuley E. Hatha Yoga Practice Improves Attention and Processing Speed in Older Adults: Results from an 8-Week Randomized Control Trial. J Altern Complement Med 2017;23:35-40.

26. Hariprasad VR, Varambally S, Shivakumar V, et al. Yoga increases the volume of the hippocampus in elderly subjects. Indian J Psychiatry 2013:55(Suppl 3):394-6.

27. Hariprasad VR, Sivakumar PT, Koparde V, et al. Effects of yoga intervention on sleep and quality-of-life in elderly: a randomized controlled trial. Indian J Psychiatry 2013;55:364-8.

28. Tulloch A, Bombell H, Dean C, et al. Yoga-based exercise improves health-related quality of life and mental well-being in older people: a systematic review of randomised controlled trials. Age Ageing 2018;47:537-44.

29. Hendriks $\mathrm{T}$, de Jong J, Cramer $\mathrm{H}$. The effects of yoga on positive mental health among healthy adults: a systematic review and metaanalysis. J Altern Complement Med 2017;23:505-17.

30. Hendriks T. The effects of Sahaja Yoga meditation on mental health: a systematic review. J Complement Integr Med 2018;15.

31. Cramer $\mathrm{H}$, Lauche R, Langhorst J, et al. Yoga for depression: a systematic review and meta-analysis. Depress Anxiety 2013;30:1068-83.

32. Cramer H, Lauche R, Anheyer D, et al. Yoga for anxiety: A systematic review and meta-analysis of randomized controlled trials. Depress Anxiety 2018;35:830-43.

33. Chen KM, Chen MH, Hong SM, et al. Physical fitness of older adults in senior activity centres after 24 -week silver yoga exercises. $J$ Clin Nurs 2008;17:2634-46.

34. Baker SP, Harvey AH. Fall injuries in the elderly. Clin Geriatr Med 1985;1:501-12.

35. Picorelli AM, Pereira DS, Felício DC, et al. Adherence of older women with strength training and aerobic exercise. Clin Interv Aging 2014;9:323-31.

36. Park CL, Groessl E, Maiya M, et al. Comparison groups in yoga research: a systematic review and critical evaluation of the literature. Complement Ther Med 2014;22:920-9.

37. Chan AW, Tetzlaff JM, Altman DG, et al. SPIRIT 2013 statement: defining standard protocol items for clinical trials. Ann Intern Med 2013;158:200-7.

38. Moher D, Hopewell S, Schulz KF, et al. CONSORT 2010 explanation and elaboration: updated guidelines for reporting parallel group randomised trials. Int J Surg 2012;10:28-55.

39. Tallberg IM, Ivachova E, Jones Tinghag K, et al. Swedish norms for word fluency tests: FAS, animals and verbs. Scand J Psychol 2008;49:479-85.

40. Greendale GA, Kazadi L, Mazdyasni S, et al. Yoga Empowers Seniors Study (YESS): Design and Asana Series. J Yoga Phys Ther 2012.2.

41. Chen KM, Tseng WS, Ting LF, et al. Development and evaluation of a yoga exercise programme for older adults. J Adv Nurs 2007;57:432-41.

42. Brassington GS, Atienza AA, Perczek RE, et al. Intervention-related cognitive versus social mediators of exercise adherence in the elderly. Am J Prev Med 2002;23(2 Suppl):80-6.

43. Rhodes RE, Martin AD, Taunton JE, et al. Factors associated with exercise adherence among older adults. An individual perspective. Sports Med 1999;28:397-411.

44. NEUGARTEN BL, Havighurst RJ, Tobin SS. The measurement of life satisfaction. J Gerontol 1961;16:134-43.

45. Wood V, Wylie ML, Sheafor B. An analysis of a short self-report measure of life satisfaction: correlation with rater judgments. $J$ Gerontol 1969;24:465-9.

46. Borg C, Fagerström C, Balducci C, et al. Life satisfaction in 6 European countries: the relationship to health, self-esteem, and social and financial resources among people (Aged 65-89) with reduced functional capacity. Geriatr Nurs 2008:29:48-57.

47. Adams DL. Analysis of a life satisfaction index. J Gerontol 1969;24:470-4

48. Diener E, Emmons RA, Larsen RJ, et al. The Satisfaction With Life Scale. J Pers Assess 1985;49:71-5.

49. Rosengren L, Jonasson SB, Brogårdh C, et al. Psychometric properties of the Satisfaction With Life Scale in Parkinson's disease. Acta Neurol Scand 2015;132:164-70.

50. Craig CL, Marshall AL, Sjöström M, et al. International physical activity questionnaire: 12 -country reliability and validity. Med Sci Sports Exerc 2003;35:1381-95.

51. Ekelund U, Sepp H, Brage S, et al. Criterion-related validity of the last 7-day, short form of the International Physical Activity Questionnaire in Swedish adults. Public Health Nutr 2006;9:258-65.

52. Rosenbaum S, Ward PB. International Working Group. The Simple Physical Activity Questionnaire. Lancet Psychiatry 2016;3:e1.

53. Berg KO, Wood-Dauphinee SL, Williams JI, et al. Measuring balance in the elderly: validation of an instrument. Can J Public Health 1992;83:7-11.

54. Conradsson M, Lundin-Olsson L, Lindelöf $\mathrm{N}$, et al. Berg balance scale: intrarater test-retest reliability among older people dependent in activities of daily living and living in residential care facilities. Phys Ther 2007;87:1155-63.

55. Tilson JK, Wu SS, Cen SY, et al. Characterizing and identifying risk for falls in the LEAPS study: a randomized clinical trial of interventions to improve walking poststroke. Stroke 2012;43:446-52.

56. Shao Z, Janse E, Visser K, et al. What do verbal fluency tasks measure? Predictors of verbal fluency performance in older adults. Front Psychol 2014;5.

57. Gottfries GG, Noltorp S, Nørgaard N. Experience with a Swedish version of the Geriatric Depression Scale in primary care centres. Int J Geriatr Psychiatry 1997;12:1029-34.

58. Yesavage JA, Brink TL, Rose TL, et al. Development and validation of a geriatric depression screening scale: a preliminary report. $J$ Psychiatr Res;17:37-49.

59. American Psychological Association. The State-Trait Anxiety Inventory (STAl). 2018 http://www.apa.org/pi/about/publications/ caregivers/practice-settings/assessment/tools/trait-state.aspx (Accessed 21 Aug 2018)

60. Lisspers J, Nygren A, Söderman E. Hospital Anxiety and Depression Scale (HAD): some psychometric data for a Swedish sample. Acta Psychiatr Scand 1997;96:281-6.

61. Berger BG, Motl RW. Exercise and mood: A selective review and synthesis of research employing the profile of mood states. J Appl Sport Psychol 2000;12:69-92.

62. Hassmén P, Blomstrand E. Mood change and marathon running: a pilot study using a Swedish version of the POMS test. Scand $J$ Psychol 1991;32:225-32.

63. Cohen S, Kamarck T, Mermelstein R. A global measure of perceived stress. J Health Soc Behav 1983;24:385-96.

64. Nordin M, Nordin S. Psychometric evaluation and normative data of the Swedish version of the 10 -item perceived stress scale. Scand $J$ Psychol 2013;54:502-7.

65. Cleeland CS. User guide for Brief Pain Inventory. 2009 https://www. mdanderson.org/documents/Departments-and-Divisions/SymptomResearch/BPI UserGuide.pdf (Accessed 8 Feb 2018).

66. MD Anderson Cancer Center. The Brief Pain Inventory. 2018 https://www.mdanderson.org/research/departments-labsinstitutes/departments-divisions/symptom-research/symptomassessment-tools/brief-pain-inventory.html (Accessed $10 \mathrm{Sep}$ 2018).

67. Bastien $\mathrm{CH}$, Vallières $\mathrm{A}$, Morin $\mathrm{CM}$. Validation of the Insomnia Severity Index as an outcome measure for insomnia research. Sleep Med 2001;2:297-307.

68. Dragioti E, Wiklund T, Alföldi P, et al. The Swedish version of the Insomnia severity index: factor structure analysis and psychometric properties in chronic pain patients. Scand J Pain 2015;9:22-7.

69. Henderson S, Duncan-Jones P, Byrne DG, et al. Measuring social relationships. The Interview Schedule for Social Interaction. Psychol Med 1980;10:723-34.

70. Hallgren M, Lundin A, Tee FY, et al. Somebody to lean on: Social relationships predict post-treatment depression severity in adults. Psychiatry Res 2017;249:261-7.

71. Borg G. Perceived exertion as an indicator of somatic stress. Scand J Rehabil Med 1970;2:92-8.

72. Cramer H, Haller H, Dobos $\mathrm{G}$, et al. A systematic review and metaanalysis estimating the expected dropout rates in randomized controlled trials on yoga interventions. Evid Based Complement Alternat Med 2016;2016:1-7. 
73. Cramer H, Ward L, Saper R, et al. The Safety of Yoga: a systematic review and meta-analysis of randomized controlled trials. Am J Epidemiol 2015;182:281-93.

74. Kinser PA, Robins JL. Control group design: enhancing rigor in research of mind-body therapies for depression. Evidence-Based Complementary and Alternative Medicine 2013;2013:1-10.
75. Harris TJ, Owen CG, Victor CR, et al. A comparison of questionnaire, accelerometer, and pedometer: measures in older people. Med Sci Sports Exerc 2009;41:1392-402. 